\title{
A study on ECG Signal Analysis and ECG databases
}

\author{
J.S.Karnewar ${ }^{1}$, Dr.V.K.Shandilya ${ }^{2}$, M.D.Tambakhe ${ }^{3}$ \\ Department of Information Technology ${ }^{1,3}$, Sipna College of Engineering \& Technology, Amravati ${ }^{1,3}$ \\ Department of Computer Science \& Engineering ${ }^{2}$, Sipna College of Engineering \& Technology, Amravati ${ }^{2}$ \\ Email: jay.skumar9@gmail.com ${ }^{1}$,vkshandilya@ rediffmail.com ${ }^{2}$
}

\begin{abstract}
The ECG (Electrocardiogram) signal is continuous in nature and abruptly changing. For taking intelligent health care decisions related with heart diseases such as paroxysmal of heart, arrhythmia diagnosing, ECG signal needs to be analyze accurately. The ECG signal basically corresponds to the electrical activity of the heart. In the literature, the ECG signal has been analyzed and utilized for various purposes, such as measuring the heart rate, examining the rhythm of heartbeats, diagnosing heart abnormalities, emotion recognition and biometric identification. ECG analysis can contain several steps, such as pre-processing, feature extraction, feature selection and classification. Performing each step is crucial for the sake of the related analysis. In this work, the literature on ECG analysis, mostly from the last decade, is comprehensively reviewed based on all of the major aspects mentioned above. Each step in ECG analysis is briefly described, and the related studies are provided. Similarly, we have focused on the ECG database used for analysis.
\end{abstract}

Keywords: ECG, Electrocardiogram, Pre-processing, Feature extraction, classification, ECG database

\section{INTRODUCTION}

The electrocardiogram (ECG) is a graphical recording of the electrical activity signals generated by the heart. The signals are generated when cardiac muscles depolarise in response to electrical impulses generated by pacemaker cells. Upon depolarisation, the muscles contract and pump blood throughout the body. The ECG is an effective noninvasive tool for various biomedical applications such as measuring the heart rate, examining the rhythm of heartbeats, diagnosing heart abnormalities, emotion recognition and biometric identification and even the approximate ischemic location in the event of a heart attack (myocardial infarction). The ECG being a nonstationary signal, the disease indicators may occur at random in the time scale. Therefore, the patient may have to be kept under observation for long intervals for accurate diagnosis. A typical ECG recording from a normal person (Fig. 1)

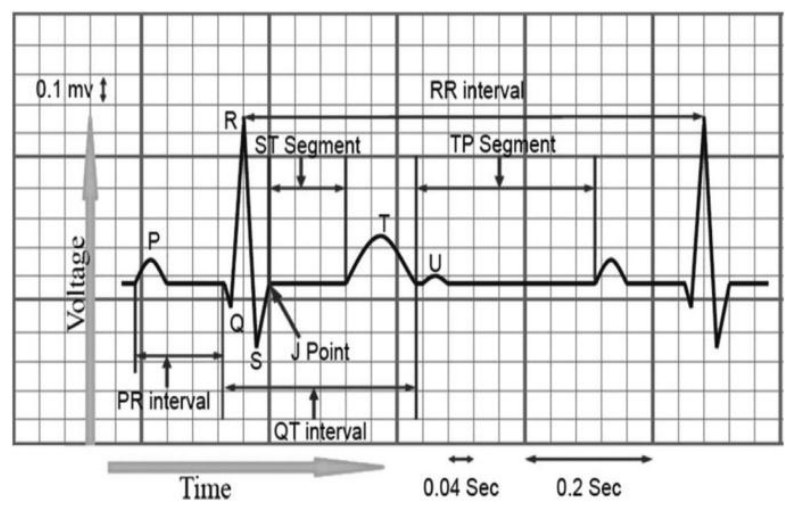

Fig.1. Standard fiducial points in the ECG signal [1].

Typical lead II ECG features and their normal values in the sinus rhythm at a heart rate of $60 \mathrm{bpm}$ for a healthy male adult [2].

\begin{tabular}{|l|c|c|}
\hline \multicolumn{1}{|c|}{ Feature } & Normal Value & $\begin{array}{c}\text { Normal } \\
\text { Limit }\end{array}$ \\
\hline P width & $110 \mathrm{~ms}$ & $\pm 20 \mathrm{~ms}$ \\
\hline PR interval & $160 \mathrm{~ms}$ & $\pm 40 \mathrm{~ms}$ \\
\hline QRS width & $100 \mathrm{~ms}$ & $\pm 20 \mathrm{~ms}$ \\
\hline $\begin{array}{l}\text { QTc (corrected) } \\
\text { interval }\end{array}$ & $400 \mathrm{~ms}$ & $\pm 40 \mathrm{~ms}$ \\
\hline P amplitude & $0.15 \mathrm{mV}$ & $\pm 0.05 \mathrm{mV}$ \\
\hline QRS height & $1.5 \mathrm{mV}$ & $\pm 0.5 \mathrm{mV}$ \\
\hline ST level & $0 \mathrm{mV}$ & $\pm 0.1 \mathrm{mV}$ \\
\hline T amplitude & $0.3 \mathrm{mV}$ & $\pm 0.2 \mathrm{mV}$ \\
\hline
\end{tabular}

Table 1: clinical features [2]

The ECG is described by waves, segments and intervals.

- Waves are labelled using the letters P, QRS, T and U. The typical normal ECG may not show a U wave.

- Segments are time durations between waves, e.g. P$R$ segment is the duration between the $P$ and $R$ waves (or $\mathrm{P}$ and $\mathrm{Q}$ waves, when $\mathrm{Q}$ wave is present).

- Intervals are time durations that include waves and segments, e.g. P-R interval is made up of the P-wave and the P-R segment.

- P-wave corresponds to the depolarisation of the atrial myocardium (muscles of upper chambers of the heart), and indicates the start of atrial contraction that pumps blood to the ventricles.

- The $\mathrm{Q}, \mathrm{R}$, and $\mathrm{S}$ waves are usually treated as a single composite wave known as the QRS complex. The QRS-complex reflects the depolarisation of ventricular myocardium, and indicates the start of ventricul contraction that pumps blood to the lungs and the rest of the body.

One of the major fields in which ECG analysis is required is the diagnosis of cardiovascular diseases. As reported by the World Health 
Organization, cardiovascular diseases are the main reason for deaths worldwide. Among the cardiovascular diseases, cardiac arrhythmias are the most common, and as a result, their precise classification has been of great interest in biomedical studies [3]. The use of ECG analysis in fields other than the diagnosis of cardiovascular diseases has also increased substantially. Many researchers have used ECG signals for emotion recognition, especially for stress level detection in addition to many other signals such as the electroencephalogram, skin temperature, blood pres-sure, electromyogram, heart rate variability, cortisol levels, and thermal imaging features. Researchers measure ECG signals at different critical moments (stress situations), such as during an oral exam, after a holiday for students, in office environments for office workers, and during a driving task for drivers. The results of these studies reveal that ECG features are useful at distinguishing the characteristics between different mental workloads and stress levels as well [1].

An ECG signal is analyzed and utilized for various purposes and applications. Depending on the application, the analysis contains several steps, such as pre-processing, feature extraction, feature selection, feature transformation and classification. Additionally, the employed success measures and appropriate constitution of the ECG databases play crucial roles.

In this paper, a comprehensive study is conducted for ECG analysis, and the subject is handled considering all the major aspects, including pre-processing, feature extraction, classification and ECG databases. Although there already exist several review articles in the literature [4, 5-7] on this topic, they are limited to only a few of these aspects rather than all. Specifically, this work comprises a survey of existing studies on ECG analysis in the literature, mostly from the last decade. For this purpose, we particularly review the articles published in journals indexed by prestigious scientific indices such as Science Citation Index and Science Citation IndexExpanded. To search the relevant studies in the literature, several platforms, such as IEEEXplore, ScienceDirect and Google Scholar, are employed, and various combinations of the keywords "electrocardiogram" and "ECG" together with "classification"; "database"; "QRS"; "feature extraction"; "feature selection" and "pre-processing" are used.

\section{PRE-PROCESSING}

ECG recordings are usually contaminated by different types of noise and artifacts. In the pre-processing step, the goals are to reduce such noise and artifacts to determine the fiducial points $(\mathrm{P}, \mathrm{Q}, \mathrm{R}, \mathrm{S}, \mathrm{T})$.

(P-onset, P-peaks, P-offset, QRS-onset, QRS-offset, T-onset, T-peaks and T-offset)) and to avoid amplitude and offset effects to compare signals from different patients. Typical types of noise are described briefly and grouped into the following categories $[3,8,9]$.

a) Power line interference: a signal in the frequency of 50 or $60 \mathrm{~Hz}$, and its bandwidth is below $1 \mathrm{~Hz}$

b) Baseline wander: a low-frequency ( 0.15 up to 0.3 $\mathrm{Hz})$ noise. This noise results from the patient inhaling and compels a baseline shifting of the ECG signals.

c) Electrode contact noise: noise that results from a deficiency in the contiguity between the electrode and skin, which adequately cuts off the measurement system from the subject.

d) Electrode motion artifacts: artifacts that result from variations in the electrode-skin impedance with electrode motion.

e) Muscle contractions (Electromyography noise): noise that results from the contraction of other muscles apart from the heart.

\subsection{Filtering}

The pre-processing stage uses a filtering block to delete artifact signals from an ECG signal [10]. Usually, an ECG signal is initially bandpass filtered with different frequency ranges before analyzing it. Bandpass filtering is widely used to delete muscle noise, baseline wander, power line interference, and low- and high-frequency noise components and to limit ADC saturation.

The frequency range of $0.1-100 \mathrm{~Hz}$ for the bandpass filtering is most often used [4]. Other frequency ranges used in band-pass filtering are 1-40 $\mathrm{Hz}$ [11-13], 0.5-40 Hz [14,15,16], 0.4-40 Hz [17], $0.05-40 \mathrm{~Hz}$ [18], 0.5-50 Hz [19], 1-120 Hz [20] and $1-100 \mathrm{~Hz}$ [21].

Analog low-pass filtering has a noticeable effect on the QRS complex, epsilon, and J-waves but does not alter the repolarization signals [22]. A good low-pass filter can filter out the noise and still leave a large amount of data for further processing [23]._A low-pass filter is designed to remove the high-frequency component in the ECG waveform. Low-pass filters with the cut-off frequency $35 \mathrm{~Hz}$ [24], $50 \mathrm{~Hz}$ [25], 100 $\mathrm{Hz}$ [26] and $70 \mathrm{~Hz}[27,28]$ were used to delete highfrequency noise and power line interference.

\section{FEATURE EXTRACTION}

Since electrocardiography is an interpretation of the electrical activity of the heart, a correct representation of the ECG signal plays an important role in the proper diagnosis of heart diseases. In the lit-erature, various feature extraction techniques have been proposed to expose the distinctive information from 
ECG signals for differ-ent purposes, such as analysis and classification. Those features can be used individually or in combination with other features. In this work, we categorize ECG features mainly into 5 groups, namely, QRS, statistical, morphological, wavelet and other features

\subsection{P-QRS-T complex features}

The P-QRS-T complex features for an ECG signal basically correspond to the locations, durations, amplitudes, and shapes of particular waves or deflections inside the signal [29,30]. Typically, an ECG signal has a total of five major deflections, including $\mathrm{P}, \mathrm{Q}, \mathrm{R}, \mathrm{S}$, and $\mathrm{T}$ waves, plus a minor deflection, namely, the $\mathrm{U}$ wave, as shown in Fig.1 [1]. The $\mathrm{P}$ wave is a small low-voltage deflection away from the baseline that is caused by the depolarization of the atria prior to atrial contraction as the activation (depolariza-tion) wave-front propagates from the sinoatrial node through the atria. The $\mathrm{Q}$ wave is a downward deflection after the $\mathrm{P}$ wave. The $\mathrm{R}$ wave follows as an upward deflection, and the $S$ wave is a down-ward deflection following the $\mathrm{R}$ wave. $\mathrm{Q}, \mathrm{R}$, and $\mathrm{S}$ waves together indicate a single event. Hence, they are usually considered to be the QRS complex. The features based on the QRS complex are among the most powerful features for ECG analysis. The QRS-complex is caused by currents that are generated when the ventricles depolarize prior to their contraction. Although atrial repolarization occurs before ventricular depolarization, the latter waveform (i.e., the QRS-complex) has a much greater amplitude, and atrial repolarization is, therefore, not seen on an ECG. The $\mathrm{T}$ wave, which follows the $\mathrm{S}$ wave, is ventricular repolarization, whereby the cardiac muscle is prepared for the next cycle of the ECG. Finally, the $\mathrm{U}$ wave is a small deflection that immediately follows the $\mathrm{T}$ wave. The $\mathrm{U}$ wave is usually in the same direction as the $\mathrm{T}$ wave.

Researchers utilize various attributes of the QRS complex as the features. Some of those attributes are the $\mathrm{R}$ wave duration, $\mathrm{P}+$ amplitude, QRS p-p amplitude, $\mathrm{R}$ wave amplitude, ST amplitude, T+ amplitude, QRS wave area and ST slope. The R wave duration is the time that passes between the beginning and end of the $\mathrm{R}$ wave [31]. The $\mathrm{P}+$ amplitude can be defined as the difference between the $\mathrm{P}$ point and the other subsequent points where the signal rises again. The QRS p-p amplitude is the difference between the $\mathrm{R}$ and $\mathrm{Q}$ points in the QRS complex in terms of the amplitude. The $\mathrm{R}$ wave amplitude can be defined as the height of the $\mathrm{R}$ wave from the base-line. The ST amplitude is the difference between the $S$ and $T$ points in terms of the amplitude values. The $\mathrm{T}+$ amplitude can be defined as the difference between the $T$ point and the subsequent point where the signal rises again. The QRS wave area is the area of the region when a rectangle is drawn on the QRS complex using the Q, R and S points [32]. The ST slope is the angle of the line, which can be drawn from the $S$ point to the $T$ point of the QRS complex. Some of the recent studies on ECG analysis that utilize QRS features are [33-39].

\section{CLASSIFICATION}

In the literature, there are various classifiers that have been utilized for ECG analysis and classification tasks. According to the recent studies reviewed in this paper, these classifiers can be mainly grouped into categories such as artificial neural networks (ANNs), LDA, $\mathrm{k}$ nearest neighbour ( $\mathrm{kNN})$, support vector machine (SVM), decision tree (DT), and Bayesian classifiers. All of these common approaches and other uncommon approaches are explained in the next subsections.

\subsection{Artificial Neural Networks (ANN)}

An ANN is a mathematical model that is inspired by biological neural networks. It includes interconnected artificial neurons, with the interconnections associated with adjustable weights; the neurons consist of input, output and/or hidden layer(s); this approach is one of the widely used pattern classifiers. ANNs aim to solve both linear classification and non-linear classification problems with various network structures and learning algorithms. The neural network structures frequently used in the ECG classification domain are as follows:

a) Complex-valued ANN: This ANN is a type of network that consists of complex-valued data, complex-number weights and complex-valued neuron activation functions [40]. Complex-valued ANNs are employed in one of the ECG classification studies [41].

b) Fuzzy clustering ANN: In this structure, a fuzzy clustering layer and an ANN layer that consists of a multilayer perceptron work sequentially. While the fuzzy layer performs the initial opera-tions for the classification task, the ANN layer works as a final classifier. Eventually, fuzzy clustering is used to improve the performance of the ANN classifier [42]. 
International Journal of Research in Advent Technology, Vol.7, No.4, April 2019

E-ISSN: 2321-9637

Available online at www.ijrat.org

\subsection{Linear Discriminant Analysis (LDA)}

LDA was originally developed in 1936 by

Fisher, and it often produces models that obtain higher classification accuracies in comparison with more modern and complex classification methods [43]. It aims to maximize the ratio of the between-class variance to the within-class variance, and it provides the highest possible discrimination between different classes. LDA is utilized in some of the recent ECG classification studies $[44,45]$.

\section{3 k nearest Neighbour $(k N N)$}

kNN classifies feature vectors according to the labels of the clos-est training samples in the feature space. For an unknown feature vector, the distances from this vector to all vectors in the training set are calculated using a distance measure such as the Euclidean distance. Then, an unknown feature vector is assigned to the class in which the closest $\mathrm{k}$ samples mostly belong to. Thus, a kind of majority voting approach is applied. The value of $\mathrm{k}$ is a positive integer and is known to be a strongly influencing factor for the accuracy of the classification. $\mathrm{kNN}$ has a wide usage in most of the pattern recognition problems and is also employed in some recent ECG classification studies [46,47].

\subsection{Support Vector Machine (SVM)}

SVM is a widely used tool for solving binary classification problems because of its outstanding generalization performance. The main idea of the SVM is to find a maximum margin between the training data and the decision boundary [48]. Support vectors, which are the training samples that are closest to the decision

\subsection{Decision Tree (DT)}

DT learning aims to map observations about an item to a con-clusion. This conclusion can be either a possible target class label or a target value. According to the difference in this conclusion, DT structures are called classification or regression trees. While the leaves of classification trees represent class labels, the leaves of regression trees represent continuous values. DT is used in some ECG classification studies [49]. In addition to common decision tree approaches, there are some more specific decision tree structures that are used frequently for ECG classification. The Random Forest Tree is a type of ensemble classifier that uses many decision trees [50]. In this approach, multiple decision trees are trained with subsets of training data. This approach uses a type of majority voting in which the output class label is assigned according to the number of votes from all the individual trees. This approach is also frequently used for ECG classification studies [51].

\subsection{Bayesian Classifier}

Bayesian classifiers are the systems that are based on Bayes' decision theory. This theory is a fundamental statistical approach [52]. The idea behind these classifiers is that if the class is known, the values of the other features can be predicted. If the class is not known, then Bayes' rule can be used to predict the class label according to the given feature values. In Bayesian classifiers, prob-abilistic models of the features are built to predict the class label of a new sample. Bayesian classifiers, which are one of the widely used methods for pattern recognition problems, are utilized in most of the recent studies [53]. The types of Bayesian classifiers utilized for ECG classification are the Bayesian network [54].

\subsection{Fuzzy based Classifier}

Apart from the abovementioned classification methods, there are also various classifiers that have been utilized for ECG classification, such as fuzzy logic classifier [55, 56] genetic fuzzy classifier [56].

\section{ECG DATABASES}

Various databases are publicly available to evaluate the methods proposed in studies that target the analysis of ECG signals. The following databases $[57,58]$ are widely used for different purposes in ECG signal analysis:

a) The Massachusetts Institute of Technology-Beth Israel Hospi-tal (MIT-BIH) Arrhythmia database is widely used for ECG signal analysis.

b) Physionet PTB Diagnostic ECG database includes 549 records from 290 persons with 52 healthy and 148 sick persons. Each sub-ject is represented by one to five records. Each record includes 15 simultaneously measured signals. The sampling rate is 1000 sam-ples/second with a 16-bit resolution over a range of $\pm 16.384 \mathrm{mV}$. The subjects were 209 men and 81 women, 17-87 years of age.

c) The QT database contains ECG recordings selected primarily from existing ECG databases, including 15 recordings from the MIT-BIH Arrhythmia Database, 6 recordings from the MIT-BIH ST Change Database, 13 recordings from the MIT-BIH Supraventricular Arrhythmia Database, 4 recordings from the MIT-BIH Long Term Database, 10 recordings from the MIT-BIH Normal Sinus Rhythm Arrhythmia Database, 33 recordings from the European Society of Cardiology ST-T 
International Journal of Research in Advent Technology, Vol.7, No.4, April 2019

E-ISSN: 2321-9637

Available online at $w w w . i j r a t . o r g$

Database, and 24 recordings from the sudden death patients gathered at Boston's Beth Israel Deaconess Medical Center. The QT Database covers a total of 105 recordings of two channel ECGs, which were chosen to prevent important baseline fluctuations or other artifacts. All the ECG signals were sampled at 250 samples/second.

d) The Apnea-ECG database contains 70 recordings with the time interval of ECG recordings ranging between 401 and $578 \mathrm{~min}$. The sampling rate is 100 $\mathrm{Hz}$. Each recording contains a continu-ous digitized ECG signal, a set of apnea annotations, and a set of machine-generated QRS.

e) Non-Invasive Fetal ECG database comprises 55 multichannel non-invasive fetal electrocardiogram recordings, collected from only one subject at 2140 weeks of pregnancy. The records have changeable durations and were collected weekly. These records can be used for testing signal separation algorithms.

f) Creighton University (CU) Ventricular Tachyarrhythmia database contains 35 eightminute (slightly less than $8.5 \mathrm{~min}$ ) single-channel ECG recordings of subjects who suffered from episodes of sustained ventricular tachycardia, ventricular flutter, and ventricular fibrillation. The sampling rate is $250 \mathrm{~Hz}$ with a 12-bit resolution over a $10-\mathrm{V}$ range.

g) The American Heart Association (AHA) database contains 80 two-channel ECG recordings. The sampling rate is $250 \mathrm{~Hz}$ per channel with a 12bit resolution over a $10-\mathrm{mV}$ range. The short version comprises five minutes of unannotated ECG prior to a thirty-minute annotated section in each recording, and the long version contains $2.5 \mathrm{~h}$ of unannotated ECG preceding each annotated segment. This database was developed for the evaluation of ventricular arrhythmia detectors.

h) Fantasia database contains records of 40 subjects. Half of them are young people between the ages of 21 and 34, and the remain-ing half are elderly people between the ages of 68 and 85 . There is a single record for each person with a two-hour interval. The sampling rate is $250 \mathrm{~Hz}$

i) The BIDMC Congestive Heart Failure database is composed of $20 \mathrm{~h}$ of ECG recordings from 15 subjects. The recordings have a sampling rate of 250 samples/second with a 12-bit resolution over a range of \pm 10 millivolts. The subjects were $11 \mathrm{men}$, 22-71 years of age, and 4 women, 54-63 years of age.

j) The European ST-T database is composed of 90 two-hour annotated ECG recordings from 79 persons. Each record has two signals; the sampling rate for each signal is $250 \mathrm{~Hz}$ with a 12-bit resolution over a nominal 20-millivolt input range. The subjects were 70 men, 30-84 years of age, and 8 women, 55-71 years of age. The ST segment and $\mathrm{T}$-wave changes were identified in both leads, and their onsets, extrema, and ends were annotated by two cardiologists.

k) The Long-Term ST database covers 86 lengthy ECG recordings of 80 persons. Each recording ranges between 21- and 24-h time intervals and is composed of two or three ECG signals. The sampling rate is $250 \mathrm{~Hz}$ with a 12-bit resolution over a range of \pm 10 millivolts.

1) The St. Petersburg Institute of Cardiological Technics (INCART) database is composed of 75 annotated recordings collected from 32 holter contacts. Each record is $30 \mathrm{~min}$ and includes 12 stan-dard leads. The sampling frequency of each record is $257 \mathrm{~Hz}$. The subjects were 17 men and 15 women, $18-80$ years of age.

The interested reader can determine detailed information on the databases mentioned in this paper at:

https://www.physionet.org/physiobank/database/\#ec g.

\section{CONCLUSIONS}

Many researchers around the world address various problems related to the analysis of ECG signals. Therefore, they need particular information on the different stages of ECG signal analysis. Although there already available many review articles in the literature on ECG analysis, they are limited to only a few aspects. In this paper, a comprehensive study has been conducted for preprocessing, feature extraction, classification, ECG databases.

Recent studies on the preprocessing step of ECG signal analysis are summarized in this paper.

In addition to the pre-processing step, the feature extraction are also very important for different tasks, such as classification or identification. Those steps are comprehensively reviewed with the related subcategories.

In the Classifier section, seven widely used classifiers, ANN, LDA, kNN, SVM, DT, Bayesian and fuzzy based classifier are reviewed in detail. Future work would cover classifiers published in the literature in view of their accordance with the databases. 
International Journal of Research in Advent Technology, Vol.7, No.4, April 2019

E-ISSN: 2321-9637

Available online at www.ijrat.org

\section{REFERENCES}

[1] H.Y. Lin, S.Y. Liang, Y.L. Ho, Y.H. Lin, H.P. Ma, Discrete-wavelet-transform-based noise removal and feature extraction for ECG signals, IRBM 35 (2014) 351-361.

[2] G.D. Clifford, F. Azuaje, P.E. McSharry, Advanced Methods and Tools for ECG Data Analysis, Artech House Publishers, 2006.

[3] R.G. Afkhami, G. Azarnia, M.A. Tinati, Cardiac arrhythmia classification using statistical and mixture modeling features of ECG signals, Pattern Recogn. Lett. 70 (2016) 45-51

[4] M. Merone, P. Soda, M. Sansone, C. Sansone, ECG databases for biometric systems: a systematic review, Expert Syst. Appl. 67 (2017) 189-202.

[5] M.S. Manikandan, S. Dandapat, Wavelet-based electrocardiogram signal compression methods and their performances: a prospective review, Biomed. Signal Process. 14 (2014) 73-107.

[6] J.D. Trigo, A. Alesanco, I. Martinez, J. Garcia, A review on digital ECG formats and the relationships between them, IEEE Trans. Inf. Technol. Biomed. 16 (2012) 432-444.

[7] E.J. Luz, W.R. Schwartz, G. Camara-Chavez, D. Menotti, ECG-based heartbeat classification for arrhythmia detection: a survey, Comput. Methods Programs Biomed. 127 (2016) 144-164.

[8] F.A. Elhaj, N. Salim, A.R. Harris, T.T. Swee, T. Ahmed, Arrhythmia recognition and classification using combined linear and nonlinear features of ECG signals, Comput. Methods Programs Biomed. 127 (2016) 52-63.

[9] G.M. Friesen, T.C. Jannett, M.A. Jadallah, S.L. Yates, S.R. Quint, H.T. Nagle, A comparison of the noise sensitivity of 9 QRS detection algorithms, IEEE Trans. Biomed. Eng. 37 (1990) 85-98.

[10]P. de Chazal, M. O’Dwyer, R.B. Reilly, Automatic classification of heartbeats using ECG morphology and heartbeat interval features, IEEE Trans. Biomed. Eng. 51 (2004) 1196-1206.

[11]F. Agrafioti, D. Hatzinakos, ECG biometric analysis in cardiac irregularity conditions, Signal Image Video Process. 3 (2009) 329-343.

[12]M.M. Tantawi, K. Revett, A.B. Salem, M.F. Tolba, A wavelet feature extraction method for electrocardiogram (ECG)-based biometric recognition, Signal Image Video Process. 9 (2015) 1271-1280.

[13]S. Gutta, Q. Cheng, Joint feature extraction and classifier design for ECG-based biometric recognition, IEEE J. Biomed. Health 20 (2016) 460-468.

[14]C. Kamath, ECG beat classification using features extracted from Teager energy functions in time and frequency domains, IET Signal Process. 5 (2011) 575-581.
[15]M.M. Tantawi, K. Revett, A. Salem, M.F. Tolba, Fiducial feature reduction analysis for electrocardiogram (ECG) based biometric recognition, J. Intell. Inf. Syst. 40 (2013) 17-39.

[16]S. Wahabi, S. Pouryayevali, S. Hari, D. Hatzinakos, On evaluating ECG biometric systems: session-dependence and body posture, IEEE Trans. Inf. Forensics Secur. 9 (2014) 2002 2013.

[17]A. Ghaffari, M.R. Homaeinezhad, M.M. Daevaeiha, High resolution ambulatory holter ECG events detection-delineation via modified multi-lead wavelet-based features analysis: detection and quantification of heart rate turbulence, Expert Syst. Appl. 38 (2011) 52995310 .

[18]T.W. Chua, W.W. Tan, Non-singleton genetic fuzzy logic system for arrhythmias classification, Eng. Appl. Artif. Intel. 24 (2011) 251-259.

[19]F.I. Donoso, R.L. Figueroa, E.A. Lecannelier, E.J. Pino, A.J. Rojas, Atrial activity selection for atrial fibrillation ECG recordings, Comput. Biol. Med. 43 (2013) 1628-1636.

[20]H. Yang, C. Kan, G. Liu, Y. Chen, Spatiotemporal differentiation of myocardial infarctions, IEEE Trans. Autom. Sci. Eng. 10 (2013) 938-947.

[21]H.H. Haseena, A.T. Mathew, J.K. Paul, Fuzzy clustered probabilistic and multi layered feed forward neural networks for electrocardiogram arrhythmia classification, J. Med. Syst. 35 (2011) 179-188.

[22]C. Watford, Understanding ECG Filtering, EMS 12-Lead, EMS-Topics, 2014.

[23]Z.C. Zhang, J. Dong, X.Q. Luo, K.S. Choi, X.J. $\mathrm{Wu}$, Heartbeat classification using disease-specific feature selection, Comput. Biol. Med. 46 (2014) 79-89.

[24]R. Alcaraz, F. Sandberg, L. Sornmo, J.J. Rieta, Classification of paroxysmal and persistent atrial fibrillation in ambulatory ECG recordings, IEEE Trans. Biomed. Eng. 58 (2011) 1441-1449.

[25]T. Castroflorio, L. Mesin, G.M. Tartaglia, C. Sforza, D. Farina, Use of electromyographic and electrocardiographic signals to detect sleep bruxism episodes in a natural environment, IEEE J. Biomed. Health 17 (2013) 994-1001.

[26]R. Alcaraz, F. Hornero, J.J. Rieta, Dynamic time warping applied to estimate atrial fibrillation temporal organization from the surface electrocardiogram, Med. Eng. Phys. 35 (2013) 1341-1348.

[27]Y. Kutlu, D. Kuntalp, A multi-stage automatic arrhythmia recognition and classification system, Comput. Biol. Med. 41 (2011) 37-45.

[28]Y. Kutlu, D. Kuntalp, Feature extraction for ECG heartbeats using higher order statistics of WPD 
International Journal of Research in Advent Technology, Vol.7, No.4, April 2019

E-ISSN: 2321-9637

Available online at $w w w . i j r a t . o r g$

coefficients, Comput. Methods Programs Biomed. 105 (2012) 257-267.

[29]Y.C. Yeh, W.J. Wang, C.W. Chiou, Cardiac arrhythmia diagnosis method using linear discriminant analysis on ECG signals, Measurement 42 (2009) 778-789.

[30]Y.C. Yeh, W.J. Wang, C.W. Chiou, Feature selection algorithm for ECG signals using RangeOverlaps Method, Expert Syst. Appl. 37 (2010) 3499-3512.

[31] L. Biel, O. Pettersson, L. Philipson, P. Wide, ECG analysis: a new approach in human identification, IEEE Trans. Instrum. Meas. 50 (2001) 808-812.

[32] P. Stravroulakis, M. Stamp, Handbook of Information and Communication Security, Springer, 2010.

[33] M. Korurek, B. Dogan, ECG beat classification using particle swarm optimization and radial basis function neural network, Expert Syst. Appl. 37 (2010) 7563-7569.

[34] P. Langley, E.J. Bowers, A. Murray, Principal component analysis as a tool for analyzing beat-tobeat changes in ECG features: application to ECGderived respiration, IEEE Trans. Biomed. Eng. 57 (2010) 821-829.

[35] S.I. Safie, J.J. Soraghan, L. Petropoulakis, Electrocardiogram (ECG) biometric authentication using pulse active ratio (PAR), IEEE Trans. Inf. Forensics Secur. 6 (2011) 1315-1322.

[36] M.R. Homaeinezhad, S.A. Atyabi, E. Tavakkoli, H.N. Toosi, A. Ghaffari, R. Ebrahimpour, ECG arrhythmia recognition via a neuro-SVM-KNN hybrid classifier with virtual QRS image-based geometrical features, Expert Syst. Appl. 39 (2012) 2047-2058.

[37] S. Ergin, A.K. Uysal, E.S. Gunal, S. Gunal, M.B. Gulmezoglu, ECG based biometric authentication using ensemble of features, 9th Iberian Conference on Information Systems and Technologies (CISTI), IEEE, Barcelona, 2014, pp. 1-6.

[38] S. Gunal, S. Ergin, E.S. Gunal, K. Uysal, ECG classification using ensemble of features, in: 47th Annual Conference on Information Sciences and Systems (CISS), IEEE, Baltimore MD, 2013, pp. $1-5$.

[39] M.R. Homaeinezhad, M. ErfanianMoshiri-Nejad, H. Naseri, A correlation analysis-based detection and delineation of ECG characteristic events using template waveforms extracted by ensemble averaging of clustered heart cycles, Comput. Biol. Med. 44 (2014) 66-75.

[40] A. Hirose, Complex-valued Neural Networks: Theories and Applications, World Scientific Publishing Company Incorporated, 2003.
[41]Y. Ozbay, A new approach to detection of ECG arrhythmias: complex discrete wavelet transform based complex valued artificial neural network, Med. Syst. 33 (2009) 435-445.

[42]R. Ceylan, Y. Ozbay, B. Karlik, A novel approach for classification of ECG arrhythmias: type-2 fuzzy clustering neural network, Expert Syst. Appl. 36 (2009) 6721-6726.

[43]Q. Liu, D. Pitt, X. Wu, On the prediction of claim duration for income protection insurance policyholders, Ann. Actuarial Sci. 8 (2014) 42-62.

[44] C.Y. Song, K.B. Liu, X. Zhang, L.L. Chen, X.C. Xian, An obstructive sleep apnea detection approach using a discriminative hidden markov model from ECG signals, IEEE Trans. Biomed. Eng. 63 (2016) 1532-1542.

[45]A. Zaknich, Principles of Adaptive Filters and Self-learning Systems, Springer Science \& Business Media, 2006.

[46]Y. Kutlu, D. Kuntalp, A multi-stage automatic arrhythmia recognition and classification system, Comput. Biol. Med. 41 (2011) 37-45.

[47]Y. Kutlu, D. Kuntalp, Feature extraction for ECG heartbeats using higher order statistics of WPD coefficients, Comput. Methods Programs Biomed. 105 (2012) 257-267.

[48] S.N. Yu, K.T. Chou, Selection of significant independent components for ECG beat classification, Expert Syst. Appl. 36 (2009) 20882096.

[49]R.N. Khushaba, S. Kodagoda, S. Lal, G. Dissanayake, Uncorrelated fuzzy neighborhood preserving analysis based feature projection for driver drowsiness recognition, Fuzzy Set Syst. 221 (2013) 90-111.

[50]R.J. Martis, U.R. Acharya, H. Prasad, C.K. Chua, C.M. Lim, J.S. Suri, Application of higher order statistics for atrial arrhythmia classification, Biomed. Signal Process. 8 (2013) 888-900.

[51]A. Jovic, N. Bogunovic, Electrocardiogram analysis using a combination of statistical, geometric, and nonlinear heart rate variability features, Artif. Intell. Med. 51 (2011) 175-186.

[52]D.L. Poole, A.K. Mackworth, Artificial Intelligence Foundations of Computational Agents, Cambridge University Press, 2010.

[53]J. Lee, D.D. McManus, P. Bourrell, L. Sornmo, K.H. Chon, Atrial flutter and atrial tachycardia detection using Bayesian approach with high resolution time-frequency spectrum from ECG recordings, Biomed. Signal Process. 8 (2013) 992 999.

[54]A. Jovic, N. Bogunovic, Electrocardiogram analysis using a combination of statistical, geometric, and nonlinear heart rate variability features, Artif. Intell. Med. 51 (2011) 175-186. 
[55]T.W. Chua, W.W. Tan, Non-singleton genetic fuzzy logic system for arrhythmias classification, Eng. Appl. Artif. Intel. 24 (2011) 251-259.

[56]M.H. Vafaie, M. Ataei, H.R. Koofigar, Heart diseases prediction based on ECG signals' classification using a genetic-fuzzy system and dynamical model of ECG signals, Biomed. Signal Process. 14 (2014) 291-296.

[57]A.L. Goldberger, L.A.N. Amaral, L. Glass, J.M. Hausdorff, P.C. Ivanov, R.G. Mark, J.E. Mietus, G.B. Moody, C.K. Peng, H.E. Stanley, PhysioBank, PhysioToolkit, and PhysioNet components of a new research resource for complex physiologic signals, Circulation 101 (2000) E215-E220.

[58] A. Alberdi, A. Aztiria, A. Basarab, Towards an automatic early stress recognition system for office environments based on multimodal measurements: a review, J. Biomed. Inform 59 (2016) 49-7 